\title{
AVALIAÇÃO BIOMECÂNICA DA INFLUÊNCIA DO MACHEAMENTO E DO DESENHO DOS PARAFUSOS CERVICAIS
}

\author{
BIOMECHANICAL EVALUATION OF THE INFLUENCE OF \\ CERVICAL SCREWS TAPPING AND DESIGN
}

Patrícia Silva', Rodrigo César. Rosa', Antonio Carlos Shimano ${ }^{2}$, Francisco José Albuquerque de Paula ${ }^{3}$, José Batista Volpon², Helton Luiz Aparecido Defino²

\section{RESUMO}

Objetivo: Avaliar a influência do desenho do parafuso (autoperfurante e automacheante) e do macheamento do orifício-piloto sobre o torque de inserção e a força de arrancamento dos parafusos utilizados para a fixação anterior da coluna cervical. Método: Quarenta parafusos automacheantes e 20 autoperfurantes foram inseridos em 10 modelos artificiais de osso (blocos de poliuretana) e 10 vértebras cervicais de carneiro. Os parâmetros estudados foram o torque de inserção e a força de arrancamento. Foram formados três grupos experimentais de acordo com o tipo de preparo do orifício- piloto e o tipo de parafuso utilizado: grupo I - parafuso automacheante com orifício- piloto perfurado e macheado; grupo II - parafuso automacheante com orifício perfurado e não macheado; grupo III - parafuso autoperfurante sem perfuração prévia do orifício- piloto e sem o macheamento. Nos grupos I e II a perfuração do orifício-piloto foi realizada por meio de broca de $3 \mathrm{~mm}$ de diâmetro e o macheamento, com $4 \mathrm{~mm}$. $\mathrm{O}$ torque de inserção foi mensurado durante a implantação dos parafusos e, em seguida, foram realizados ensaios mecânicos em máquina universal de testes para avaliar a força de arrancamento dos parafusos. Resultados: O macheamento e a perfuração do orifício- piloto reduziram significativamente o torque de inserção e a força de arrancamento. Conclusão: $\mathrm{O}$ torque de inserção e a força de arrancamento dos parafusos autoperfurantes foram significativamente maiores quando comparado com os dos parafusos automacheantes inseridos após o macheamento do orifício-piloto.

Descritores - Coluna vertebral; Parafusos ósseos; Dispositivos de fixação ortopédica; Biomecânica

\section{ABSTRACT}

Objective: To assess if the screw design (self-drilling/selftapping) and the pilot hole tapping could affect the insertion torque and screw pullout strength of the screw used in anterior fixation of the cervical spine. Methods: Forty self-tapping screws and 20 self-drilling screws were inserted into 10 models of artificial bone and 10 cervical vertebrae of sheep. The studied parameters were the insertion torque and pullout strength. The following groups were created: Group I - selftapping screw insertion after pilot hole drilling and tapping; Group II - self-tapping screw insertion after pilot hole drilling without tapping; Group III - self-drilling screw insertion without drilling and tapping. In Groups I and II, the pilot hole had $14.0 \mathrm{~mm}$ in depth and was made with a 3mmn drill, while tapping was made with a $4 \mathrm{~mm}$ tap. The insertion torque was measured and the pullout test was performed. The comparison between groups was made considering the mean insertion torque and the maximum mean pullout strength with the variance analysis (ANOVA; $p \leq 0.05)$. Results: Previous drilling and tapping of pilot hole significantly decreased the insertion torque and the pullout strength. Conclusion: The insertion torque and pullout strength of self-drilling screws were significantly higher when compared to self-tapping screws inserted after pilot hole tapping.

Keywords - Spine; Bone screw; Orthopedic fixation devices; Biomechanics

1 - Pós-graduando da Faculdade de Medicina da Universidade de São Paulo-USP - Ribeirão Preto (SP), Brasil.

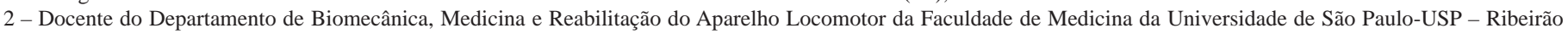
Preto (SP), Brasil

3 - Docente do Departamento de Medicina Interna da Faculdade de Medicina da Universidade de São Paulo-USP - Ribeirão Preto (SP), Brasil.

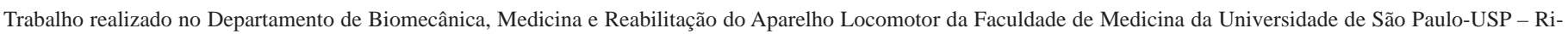
beirão Preto, com apoio Fapesp e Capes-Probral.

Correspondência: Helton L.A. Defino, Rua Dornélia de Souza Mosca, 235, Jardim Canadá - 14024-120 - Ribeirão Preto (SP), Brasil.

E-mail: hladefin@fmrp.usp.br 


\section{INTRODUÇÃO}

As placas anteriores para a fixação da coluna cervical têm sido utilizadas para estabilizar o segmento cervical durante o processo de consolidação do enxerto ósseo das artrodeses ${ }^{(1)}$. Os benefícios adicionais do uso de placa e parafusos estão bem estabelecidos ${ }^{(2,3)}$.

A estabilização mecânica proporcionada pelo sistema de placas está relacionada com vários fatores, destacando-se entre eles a ancoragem dos parafusos no tecido ósseo ${ }^{(3)}$. A densidade mineral óssea é o principal fator que interfere na estabilidade mecânica do implante. Entretanto, esse é um dos parâmetros que não podem ser controlados pelo cirurgião, de modo que os novos desenhos de parafusos têm sido desenvolvidos para melhorar a qualidade da fixação.

Os parafusos de fixação bicortical ou unicortical da placa cervical anterior foram desenvolvidos para ser inseridos nos orifício-piloto previamente perfurados e macheados. A utilização dos parafusos unicorticais tornou o procedimento cirúrgico mais simples e reduziu os riscos, quando comparados com os da fixação bicortical $^{(5,6)}$

Entretanto, a perfuração e o macheamento do orifício-piloto resultam em trauma adicional e aumento do tempo operatório para a realização dos procedimentos. Por essa razão, mudanças nos desenhos dos parafusos foram realizadas com o intuito de eliminar o macheamento e a perfuração do orifício-piloto; foram desenvolvidos os parafusos automacheantes e os autoperfurantes. A vantagem no uso desses parafusos é que podem ser inseridos sem a realização prévia do macheamento ou fixados diretamente no osso, simplificando o procedimento cirúrgico.

Porém, mudanças no desenho dos parafusos podem causar repercussão no seu desempenho mecânico, sendo necessários estudos comparando o desempenho dos novos desenhos de parafusos para estabelecer as reais vantagens e desvantagens de cada modelo.

O macheamento do orifício-piloto tem sido tema controvertido no âmbito da literatura que aborda este tema. Esse procedimento é utilizado para preparar o tecido ósseo adjacente para a introdução do parafuso.

O desenvolvimento mecânico agudo dos parafusos pode ser avaliado por meio do torque e da resistência ao arrancamento dos implantes. O torque de inserção é definido como o momento angular de força exigida para avançar o parafuso no interior do material de fi- xação e está diretamente relacionado com a qualidade do sistema de fixação.

O objetivo do trabalho foi estudar a influência do desenho do parafuso (autoperfurante e automacheante) e do macheamento do orifício-piloto sobre o torque de inserção e a resistência ao arrancamento dos parafusos utilizados para a fixação anterior da coluna cervical.

\section{MÉTODO}

Foram utilizados no estudo 40 parafusos automacheantes e 10 parafusos autoperfurantes, ambos de titânio, utilizados na fixação da placa cervical (CSLP - Synthes ${ }^{\circledR}$ ). Os parafusos possuem cabeça expansora, $4 \mathrm{~mm}$ de diâmetro externo e $14 \mathrm{~mm}$ de comprimento (Figura 1).

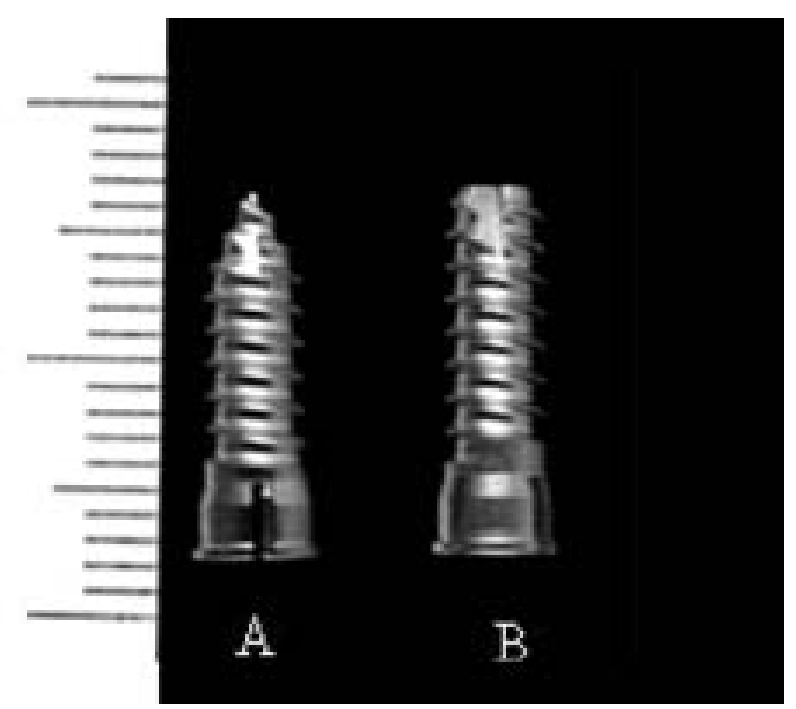

Figura 1 - Parafusos utilizados no estudo. A) parafuso autoperfurante e B) parafuso automacheante (CSLP Synthes ${ }^{\circledR}$ )

O torque de inserção e a resistência ao arrancamento foram mensurados com a inserção dos parafusos no corpo cervical anterior das vértebras de carneiro. Foram utilizadas dez vértebras cervicais (C3-C6) de carneiros da raça Santa Inês Deslanadados com média de idade de $12 \pm 3$ meses. Após sua retirada, foi removido o tecido muscular, examinado e sua densidade mineral óssea foi mensurada pelo dual-energy $X$-ray absorptiometry (DEXA®) usando o $Q D R$ system com software versão 11 - 2:5 (Hologic $4500 W \AA$, Waltham, MA, EUA). As vértebras com densidade mineral óssea média de $0,33 \pm 0,01 \mathrm{~g} / \mathrm{cm}^{3}\left(0,32-0,34 \mathrm{~g} / \mathrm{cm}^{3}\right)$ foram selecionadas para o estudo. 
Em cada vértebra cervical foram testados três parafusos, cada um correspondendo a um grupo experimental. Os parafusos foram inseridos, aproximadamente, a 5mm de distância ente si para evitar possível interferência na mensuração do torque de inserção e da força de arrancamento.

Os grupos experimentais foram formados de acordo com a técnica de preparo do orifício-piloto e o tipo de parafuso utilizado no estudo (automacheante e autoperfurante): grupo I - parafuso automacheante inserido após a perfuração e o macheamento do orifício-piloto; grupo II - parafuso automacheante inserido após a perfuração do orifício-piloto sem o macheamento; grupo III - parafuso autoperfurante inserido diretamente nos corpos vertebral sem a realização da perfuração e nem do macheamento.

O orifício-piloto (grupos I e II) foi confeccionado por meio de broca com 3,0mm de diâmetro e perfurado com 14mm de profundidade. Os parafusos foram inseridos no corpo anterior da vértebra cervical simulando a sua utilização clínica.

No grupo II, o macheamento foi realizado utilizando macho com 4mm de diâmetro para cortar e preparar o tecido ósseo para a inserção do parafuso.

O torque de inserção dos implantes foi mensurado utilizando microtorquímetro digital da marca $M K^{\circledR}$, modelo TI-500/MKMT-1, 1N.m com capacidade de resolução de 0,001N.m e foi utilizado o software Graphic III ${ }^{\circledR}$ para a análise dos dados.

Os ensaios mecânicos foram realizados utilizando máquina universal de ensaio Emic ${ }^{\circledR}$ com célula de carga com capacidade de $1.000 \mathrm{~N}$ e os dados foram analisados por meio do software Tesc $3.13^{\circledR}$.

Para a realização dos ensaios mecânicos de arrancamento, a cabeça do parafuso foi fixada à máquina de teste por meio de conectores que permitiam movimentos multidirecionais e aplicação de carga axial de tração sem a inserção de torque. Pré-carga de $5 \mathrm{~N}$ foi aplicada por um período de 10 segundos para acomodação do sistema; então, carga axial de tração foi aplicada na constante de $0,2 \mathrm{~mm} / \mathrm{min}$ até o arrancamento do implante (Figura 2).

Dez ensaios mecânicos e 10 medidas de torque de inserção foram realizados para cada grupo experimental. No total, 30 análises de torque e 30 testes mecânicos foram realizados.

Os resultados foram submetidos ao teste de análise de variância multifatorial (ANOVA) e, pelo teste de comparações múltiplas de Tukey-Kramer, foi estabelecido nível de significância de 5\% $(\mathrm{p} \leq 0,05)$.

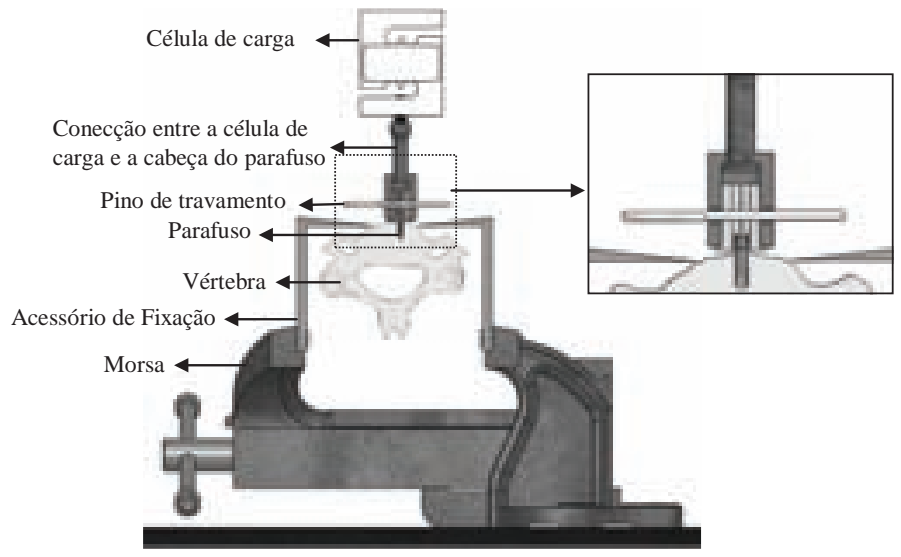

Figura 2 - Desenho esquemático dos acessórios utilizados nos ensaios mecânicos. Em destaque: visão mais nítida do parafuso e dos acessórios utilizados para sua fixação.

\section{RESULTADOS}

\section{Torque de inserção}

A média máxima do torque de inserção dos parafusos implantados na vértebra cervical de carneiro e no modelo artificial de osso nos grupos de comparação está apresentada na Tabela 1 e Figura 3.

Tabela 1 - Valores médios e desvio padrão do torque médio máximo nos três grupos experimentais no modelo artificial de osso e nas vértebras cervicais e comparação entre os grupos

\begin{tabular}{|c|c|c|c|}
\hline & Grupos experimentais & $\begin{array}{c}\text { Torque de } \\
\text { inserção (N.m) }\end{array}$ & $\begin{array}{l}\text { Comparação entre } \\
\text { os grupos } \\
\text { (p value) }\end{array}$ \\
\hline \multirow{3}{*}{$\begin{array}{l}\text { Modelo } \\
\text { artificial de } \\
\text { osso } \\
\text { (blocos de } \\
\text { poliuretana) }\end{array}$} & $\begin{array}{l}\text { I-Perfurado e macheado } \\
\text { (CSLP automacheante) }\end{array}$ & $0,04 \pm 0,01$ & $\begin{array}{c}|x| \mid \\
p<0,001\end{array}$ \\
\hline & $\begin{array}{l}\text { II-Perfurado e não macheado } \\
\text { (CSLP automacheante) }\end{array}$ & $0,08 \pm 0,01$ & $\begin{array}{c}\|x\| \| \\
p<0,001\end{array}$ \\
\hline & $\begin{array}{l}\text { III - Não perfurado e não macheado } \\
\text { (CSLP autoperfurante) }\end{array}$ & $0,27 \pm 0,03$ & $\begin{array}{c}|\| x| \\
p<0,001\end{array}$ \\
\hline \multirow{3}{*}{$\begin{array}{l}\text { Vértebras } \\
\text { cenvicais }\end{array}$} & $\begin{array}{l}\text { I-Perfurado e macheado } \\
\text { (CSLP automacheante) }\end{array}$ & $0,05 \pm 0,01$ & $\begin{array}{c}|x| \mid \\
p<0,001\end{array}$ \\
\hline & $\begin{array}{l}\text { II- Perfurado e não macheado } \\
\text { (CSLP automacheante) }\end{array}$ & $0,16 \pm 0,02$ & $\begin{array}{c}\|x\| I \\
p<0,001\end{array}$ \\
\hline & $\begin{array}{l}\text { III - Não perfurado e não macheado } \\
\text { (CSLP autoperfurante) }\end{array}$ & $0,33 \pm 0,03$ & $\begin{array}{c}|||x| \\
p<0,001\end{array}$ \\
\hline
\end{tabular}

\section{Força de arrancamento}

A média máxima da força de arrancamento dos parafusos implantados na vértebra cervical de carneiro e modelo artificial de osso nos grupos de comparação está representada na Tabela 2 e Figura 4. 

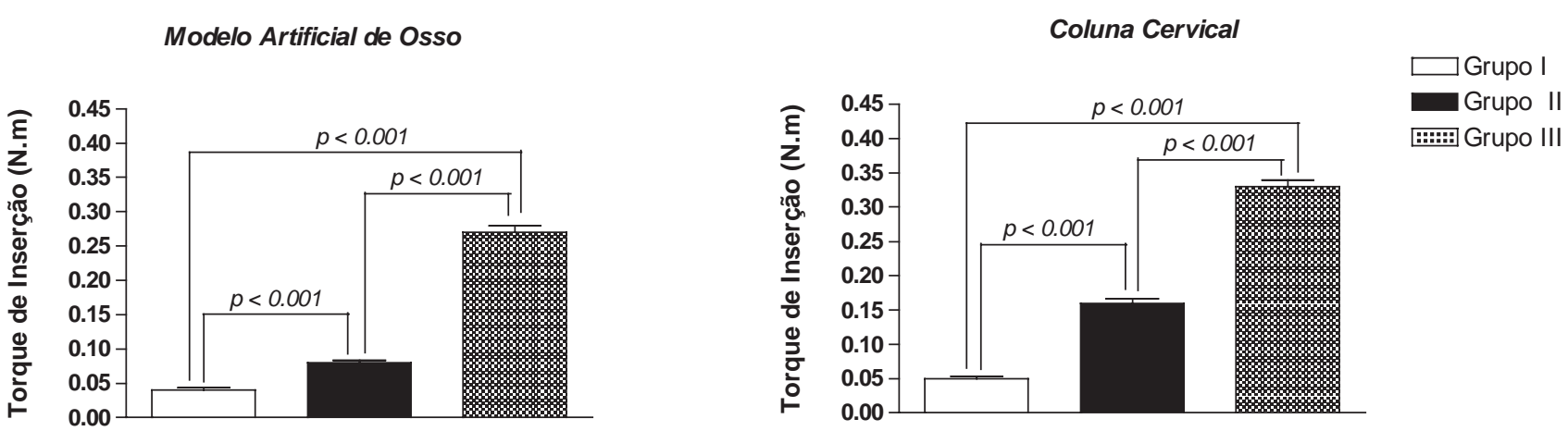

Figura 3 - Comparação entre os valores médios máximos do torque de inserção nos três grupos experimentais dos parafusos inseridos no modelo artificial de osso e na vértebra cervical e comparação entre os grupos

Tabela 2 - Valores médios e desvio padrão da força média máxima de arrancamento nos três grupos experimentais no modelo artificial de osso e nas vértebras cervicais e comparação entre os grupos

\begin{tabular}{|c|c|c|c|}
\hline & Grupo experimental & $\begin{array}{c}\text { Força de } \\
\text { arrancamento } \\
\text { (N) }\end{array}$ & $\begin{array}{c}\text { Comparação } \\
\text { entre os grupos } \\
\text { ( } p \text { value) }\end{array}$ \\
\hline \multirow{3}{*}{$\begin{array}{l}\text { Modelo } \\
\text { artificial de } \\
\text { osso } \\
\text { (blocos de } \\
\text { poliuretana) }\end{array}$} & $\begin{array}{l}\text { I - Perfurado e macheado } \\
\text { (CSLP automacheante) }\end{array}$ & $368,90 \pm 23,48$ & $\begin{array}{c}|x| \mid \\
p<0,05\end{array}$ \\
\hline & $\begin{array}{l}\text { II - Perfurado e não macheado } \\
\text { (CSLP automacheante) }\end{array}$ & $393,16 \pm 18,71$ & $\begin{array}{c}\|x\| \| \\
p<0,001\end{array}$ \\
\hline & $\begin{array}{l}\text { III - Não perfurado e não macheado } \\
\text { (CSLP autoperfurante) }\end{array}$ & $449,12 \pm 14,79$ & $\begin{array}{c}|||\times| \\
p<0,001\end{array}$ \\
\hline \multirow{3}{*}{$\begin{array}{l}\text { Vértebra } \\
\text { cervical }\end{array}$} & $\begin{array}{l}\text { I-Perfurado e macheado } \\
\text { (CSLP automacheante) }\end{array}$ & $237,12 \pm 24,97$ & $\begin{array}{c}|x| \mid \\
p<0,05\end{array}$ \\
\hline & $\begin{array}{l}\text { II - Perfurado e não macheado } \\
\text { (CSLP automacheante) }\end{array}$ & $308,89 \pm 80,63$ & $\begin{array}{c}\|x\| \mathrm{II} \\
p<0,05\end{array}$ \\
\hline & $\begin{array}{l}\text { III - Não perfurado e não macheado } \\
\text { (CSLP autoperfurante) }\end{array}$ & $381,95 \pm 53,46$ & $\begin{array}{c}|||\times| \\
p<0,001\end{array}$ \\
\hline
\end{tabular}

\section{DISCUSSÃO}

Este estudo procurou investigar e sanar dúvidas referentes às características biomecânicas dos novos desenhos de parafusos que foram especialmente projetados para a fixação cervical ${ }^{(8,9)}$. O nosso estudo foi projetado visando estabelecer comparação biomecânica dos parafusos autoperfurantes e automacheantes. Além disso, incluímos outro grupo no qual o parafuso automacheante foi inserido no orifício-piloto previamente macheado. Esse último grupo foi criado baseado nas circunstâncias clínicas práticas. Em algumas situações cirúrgicas, depois de inserido o parafuso no orifício-piloto previamente macheado poderia haver a necessidade de trocar o implante, principio aplicado para o parafuso de salvamento. É possível inserir um parafuso automacheante no orifício-piloto previamente macheado e obter boa qualidade de fixação? Esse evento também pode ocorrer com o uso do orifício promovido pela aplicação do fixador temporário da placa cervical.
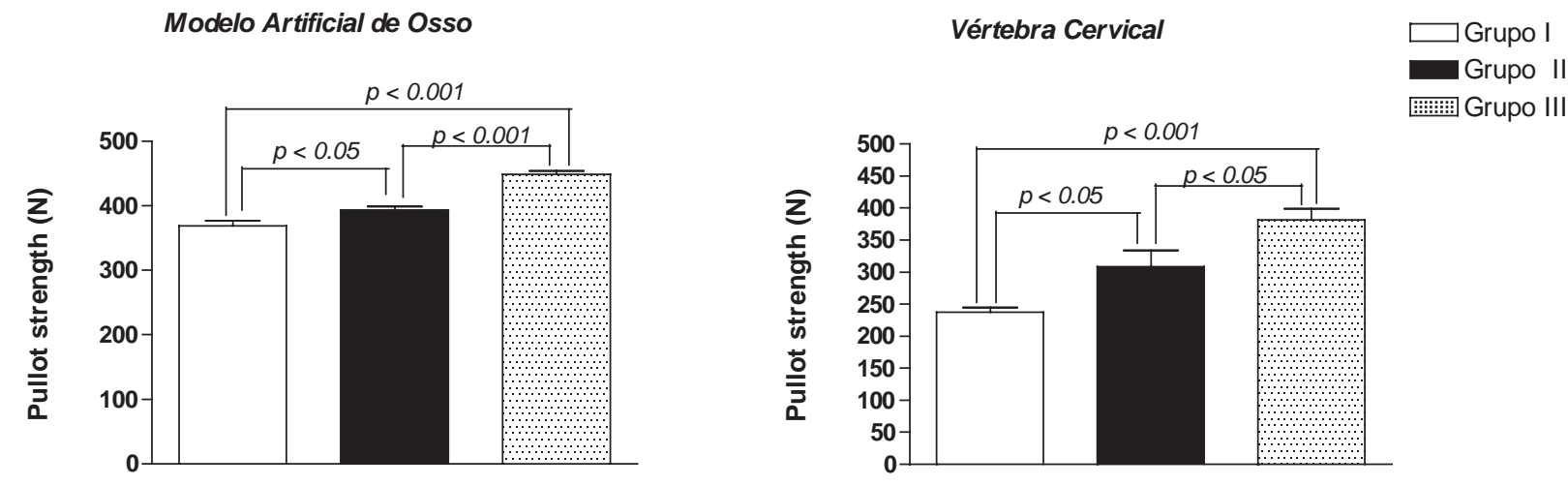

Figura 4 - Comparação entre os valores médios máximos da força de arrancamento nos três grupos experimentais dos parafusos inseridos no modelo artificial de osso e na vértebra cervical e comparação entre os grupos 
Os resultados mostraram que o torque de inserção e a força de arrancamento possuem relevância clínica significativamente diferente para os três grupos. A melhor qualidade da fixação foi obtida com o uso dos parafusos autoperfurantes e os piores resultados da fixação foram observados quando o parafuso automacheante foi inserido no orifício-piloto previamente macheado. Isso mostra claramente o efeito negativo do macheamento do orifício-piloto. Na comparação do orifício-piloto macheado e não macheado, observamos redução do torque de inserção e da resistência ao arrancamento, quando o orifício-piloto foi previamente macheado, que corrobora o verificado por outros autores ${ }^{(10,11)}$.

O estudo demonstrou que o uso do parafuso autoperfurante na prática clínica, além de reduzir o tempo e o número de procedimentos cirúrgicos, proporciona maior fixação do sistema quando inserido no tecido ósseo não osteoporótico ou osteopênico. A densidade mineral óssea das vértebras humanas normais está bem reportada na literatura e varia em média de 0,30 a $0,34 \mathrm{~g} / \mathrm{cm}^{3(12,13)}$. As vértebras cervicais de carneiros utilizadas no presente estudo correspondem à densidade das vértebras humanas não osteoporóticas, com densidade média de $0,33 \pm 0,01 \mathrm{~g} / \mathrm{cm}^{3}\left(0,32-0,34 \mathrm{~g} / \mathrm{cm}^{3}\right)$.

Contudo, Hitchon et al ${ }^{(14)}$ não observaram diferença na força de arrancamento na comparação entre os parafusos autoperfurantes e automacheantes com as mesmas geometrias dos utilizados no nosso estudo. Neste estudo, o grupo dos parafusos autoperfurantes apresentou maior resistência ao arrancamento quando comparado com o dos parafusos automacheantes similares, mas essa diferença não foi estatisticamente significante. Os autores utilizaram no estudo vértebras cervicais humanas em situações que não refletem as reais condições do osso humano, por apresentarem grande variação e qualidade da densidade mineral óssea. Contudo, as vantagens encontradas em nosso estudo favorecem a utilização dos parafusos automacheantes ou autoperfurantes, que podem ser contrabalançadas nas situações em que o osso se apresenta osteoporótico. Pensamos que essa hipótese deveria ser investigada no futuro, mas o estudo de Conrad et al ${ }^{(15)}$ não observou nenhuma diferença na força de arrancamento dos parafusos automacheantes e autoperfurantes usando blocos de poliuretana com densidade comparável à dos ossos osteoporóticos.

O aumento da força de arrancamento dos parafusos autoperfurantes é apoiada pelo fato de a resistência ao arrancamento ser proporcional ao volume de osso entre as roscas $^{(16)}$. Foi observado experimentalmente que a interface parafuso/osso dos parafusos autoperfurantes era superior à dos parafusos automacheantes e não causaria danos ao tecido ósseo adjacente ao implante ${ }^{(17)}$.

\section{CONCLUSÃO}

Observamos diferença no torque de inserção e na resistência ao arrancamento na comparação entre os parafusos automacheantes e dos autoperfurantes fixados no modelo artificial de osso e na vértebra cervical de carneiro.

Os parafusos autoperfurantes apresentaram maiores valores médios de torque de inserção e força de arrancamento quando comparados com os dos parafusos automacheantes.

O macheamento do orifício-piloto promove significante redução do torque de inserção e da força de arrancamento.

\section{AGRADECIMENTO}

Trabalho realizado com o apoio do CNPq ( Conselho Nacional de Desenvolvimento Científico e Tecnológico).

\section{REFERÊNCIAS}

1. Dvorak MF, Pitzen T, Zhu Q, Gordon JD, Fisher CG, Oxland TR. Anterior cervical plate fixation: a biomechanical study to evaluate the effects of plate design, endplate preparation, and bone mineral density. Spine. 2005;30(3):294-301.

2. Wang JC, McDonough PW, Endow KK, Delamarter RB. Increased fusion rates with cervical plating for two-level anterior cervical discectomy and fusion. Spine. 2000;25(1):41-5

3. Wang JC, McDonough PW, Kanim LE, Endow KK, Delamarter RB. Increased fusion rates with cervical plating for three-level anterior cervical discectomy and fusion. Spine. 2001;26(6):643-6.

4. Öktenoglu BT, Ferrara LA, Andalkar N, Özer AF, Sarioglu AC, Benzel EC. Effects of hole preparation on screw pullout resistance and insertional torque: a biomechanica study. J Neurosurg. 2001;94(1 Suppl):91-6.

5. Kostuik JP, Connolly PJ, Esses SI, Suh P. Anterior cervical plate fixation with the titanium hollow screw plate system. Spine. 1993;18(10):1273-8.

6. Suh PB, Kostuik JP, Esses SI. Anterior cervical plate fixation with the titanium hollow screw plate system. A preliminary report. Spine. 1990;15(10):1079-81.

7. Yang S, Wang LW. Biomechanical comparison of the stable efficacy of two anterior plating systems. Clin Biomech.(Bristol, Avon). 2003;18(6):S59-S66.

8. Conrad BP, Cordista AG, Horodyski M, Rechtine GR. Biomechanical evaluation of the pullout strength of cervical screws. J Spinal Disord Tech. 2005;18(6):506-10.

9. Hitchon PW, Brenton MD, Coppes JK, From AM, Torner JC. Factors affecting the pullout strength of self-drilling and self-tapping anterior cervical screws. Spine. 2003;28(1):9-13

10. Chapman JR, Harrington RM, Lee KM, Anderson PA, Tencer AF, Kowalski D. Factors affecting the pullout strength of cancellous bone screws. J Biomech Eng. 1996;118(3):391-8.

11. Reitman CA, Nguyen L, Fogel GR. Biomechanical evaluation of relationship of screw pullout strength, insertional torque, and bone mineral density in the cervical spine. J Spinal Disord Tech. 2004;17(4):306-11.

12. Hsu CC, Chao CK, Wang JL, Hou SM, Tsai YT, Lin J. Increase of pullout strength of spinal pedicle screws with conical core: biomechanical tests and finite element analyses. J Orthop Res. 2005;23(4):788-94.

13. Kandziora F, Pflugmacher R, Scholz M, Schnake K, Lucke M, Schroder R, et al. Comparison between sheep and human cervical spines: an anatomic, radiographic, bone mineral density, and biomechanical study. Spine. 2001;26(9):1028-37.

14. Hitchon PW, Brenton MD, Coppes JK, From AM, Torner JC. Factors affecting the pullout strength of self-drilling and self-tapping anterior cervical screws. Spine. 2003;28(1):9-13.

15. Conrad BP, Cordista AG, Horodyski M, Rechtine GR. Biomechanical evaluation of the pullout strength of cervical screws. J Spinal Disord Tech. 2005;18(6):506-10.

16. Benzel EC. Implant-bone interfaces. In: Benzel EC, editor. Biomechanics of spinal stabilization. New York: Thieme; 2001. p.155-70.

17. Reitman CA, Nguyen L, Fogel GR. Biomechanical evaluation of relationship of screw pullout strength, insertional torque, and bone mineral density in the cervical spine. J Spinal Disord Tech. 2004;17(4):306-11. 